\title{
ENTRENAMIENTO EN MINDFULNESS PARA PACIENTES CON TRASTORNO POR DÉFICIT DE ATENCIÓN CON HIPERACTIVIDAD (TDAH): UNA REVISIÓN DESCRIPTIVA
}

\section{MINDFULNESS TRAINING FOR PATIENTS WITH ATTENTION-DEFICIT/HYPERACTIVITY DISORDER (ADHD): A DESCRIPTIVE REVIEW}

\section{Eduard Forcadell López}

Institut de Neuropsiquiatria i Addicions, Hospital del Mar, Barcelona, España.

Departamento de Psiquiatría y Medicina Legal, Instituto de Neurociencias, Facultad de Medicina, Universidad Autónoma de Barcelona, Bellatera, España.

\section{Mònica Astals Vizcaino}

Institut de Neuropsiquiatria i Addicions, Hospital del Mar, Barcelona, España.

\section{Devi Treen Calvo}

Institut de Neuropsiquiatria i Addicions, Hospital del Mar, Barcelona, España.

\section{Jacobo Chamorro López}

Institut de Neuropsiquiatria i Addicions, Hospital del Mar, Barcelona, España.

\section{Santiago Batlle Vila}

Institut de Neuropsiquiatria i Addicions, Centre de Salut Mental Infanto-Juvenil Sant Martí - La Mina, Parc de Salut Mar, Barcelona, España.

Cómo referenciar este artículo/How to reference this article:

Forcadell López, E., Astals Vizcaino, M., Treen Calvo, D., Chamorro López, J. y Batlle Vila, S. (2016). Entrenamiento en Mindfulness para Pacientes con Trastorno por Déficit de Atención con Hiperactividad (TDAH): una Revisión Descriptiva. Revista de Psicoterapia, 27(103), 203-213.

Fecha de recepción: 8/11/2015. Fecha de aceptación v1: 3/02/2016. Fecha de aceptación v2: 11/02/2016 Correspondencia sobre este artículo:

E-mail:60761@parcdesalutmar.cat

Dirección postal: Eduard Forcadell López, Institut de Neuropsiquiatria i Addicions, Hospital del Mar. Paseo marítimo 25-29, Barcelona 08003 (España). 


\title{
Resumen
}

Introducción y Objetivos: El trastorno por déficit de atención con hiperactividad (TDAH) es uno de los trastornos del neurodesarrollo más frecuentes en la infancia y a menudo persiste en la edad adulta. Los tratamientos conductuales y farmacológicos constituyen las intervenciones más eficaces. Para paliar algunos de sus déficits ha surgido un interés en nuevos tratamientos. El mindfulness (atención plena) se ha posicionado como una aproximación prometedora para abordar sintomas nucleares del trastorno y otras afecciones relacionadas. El objetivo del presente trabajo es realizar una revisión descriptiva para investigar la evidencia de los tratamientos basados en mindfulness para personas con TDAH.

Método: Se realizó una búsqueda en la base de datos electrónica Pubmed, utilizando las palabras clave "mindfulness", "ADHD”, "attention deficit disorder with Hyperactivity”. Los criterios de inclusión fueron: articulos en inglés que emplearan intervenciones basadas en mindfulness para tratar pacientes (niños y adultos) con TDAH. Siete publicaciones cumplieron los criterios de selección para formar parte de la muestra.

Resultados: Se obtuvieron resultados positivos en todos los trabajos en referencia a la disminución de la sintomatología atencional e hiperactiva/impulsiva (en algunos casos reducciones superiores al 30\% de los síntomas) medida a través de autoinformes. Los resultados en tareas neuropsicológicas no fueron tan favorables ni consistentes.

Discusión: Las intervenciones basadas en mindfulness para tratar pacientes con TDAH han mostrado resultados prometedores. En futuras investigaciones será necesario incorporar mejoras metodológicas; incrementar el tamaño de las muestras, grupos control, evaluaciones clínicas, incluir pruebas neuropsicológicas y examinar los efectos a largo plazo del tratamiento.

Palabras clave: TDAH, mindfulness, entrenamiento, revisión.

\begin{abstract}
Introduction and Objectives: The attention deficit hyperactivity disorder (ADHD) is one of the most common neurodevelopmental disorders in childhood and often persists into adulthood. Behavioral and pharmacological treatments are the most effective interventions. To overcome some of their deficiencies there has been an interest in new treatments. Mindfulness has emerged as a promising approach to address core symptoms of the disorder and other related conditions. The aim of this work is to perform a descriptive review to investigate evidence of mindfulness-based treatments for people with ADHD.

Method: A search was performed in Pubmed electronic database, using the keywords "mindfulness", "ADHD”, "Attention Deficit Disorder with Hyperactivity". Inclusion criteria were: articles in English that study mindfulness-based treatments for patients (children and adults) with ADHD. Seven publications met the criteria to be part of the final sample.

Results: Positive results were obtained in all the works referring to the reduction of attentional and hyperactive/impulsive symptoms (in some cases reductions over $30 \%$ of symptoms) as measured by self-report. The results obtained with neuropsychological tasks were not as favorable or consistent.

Discussion: Mindfulness-based interventions to treat ADHD patients have shown promising results. Future research will need to incorporate methodological improvements; increase the size of the samples, control groups, clinical evaluations, neuropsychological tests and examine the long-term effects of treatment.
\end{abstract}

Keywords: $A D H D$, mindfulness, mindfulness-based interventions, review. 


\section{Introducción}

El trastorno por déficit de atención con hiperactividad (TDAH) es uno de los trastornos neuropsiquiátricos más frecuentes en la infancia y a menudo persiste en la edad adulta (Barkley, 1997). El diagnóstico requiere síntomas atencionales y/o conducta hiperactiva/impulsiva que causen una interferencia en el funcionamiento social, académico u ocupacional. No obstante, los déficits en las funciones ejecutivas y en la regulación emocional también están presentes en muchos pacientes (Martel, 2009) (Marije, Oosterlaan, Sergeant y Buitelaar, 2005). Los tratamientos conductuales y farmacológicos (principalmente estimulantes) son los tratamientos más eficaces para el TDAH como lo demuestran numerosos ensayos clínicos aleatoritzados (Biederman, 2004). Sin embargo, ha surgido un interés en nuevas intervenciones no farmacológicas para optimizar los resultados en aquellos pacientes que no responden a la farmacoterapia (o lo hacen parcialmente), o para paliar algunos de los déficits de estos tratamientos, como el funcionamiento únicamente a corto plazo, los efectos secundarios y la baja adherencia (Schachter, Pham, King, Langford y Moher, 2001). Por otra parte, es necesario ampliar el abanico de intervenciones más allá de los síntomas centrales del TDAH para abordar aspectos ya mencionados como la disregulación emocional y los déficits en las funciones ejecutivas.

En este sentido, el mindfulness (atención plena) ha surgido como una nueva aproximación prometedora para tratar los síntomas principales y las afectaciones relacionadas en personas con este trastorno. El mindfulness (o atención plena) consiste en la práctica formal del aumento de la conciencia, la atención en el momento presente y la observación sin prejuicios (Kabat-Zinn, 2003). Se ha conceptualizado como un modelo de dos componentes que consiste en (a) orientar la atención en el momento presente y (b) aproximarse a la propia experiencia con curiosidad, apertura y aceptación (Bishop et al., 2004). Intervenciones basadas en mindfulness han demostrado eficacia en pacientes que sufren depresión, estrés y dolor (Baer, 2003; Hofmann, Sawyer, Witt y Oh, 2010). El entrenamiento en mindfulness puede ser una opción viable para tratar el TDAH como lo demuestra la numerosa evidencia aportada en diversas áreas relacionadas con el trastorno. La investigación reciente ha demostrado que el entrenamiento en atención plena puede modificar el funcionamiento de las redes atencionales (Jha, Krompinger y Baime, 2007), mejora el rendimiento en tareas que miden funciones ejecutivas (atención, memoria de trabajo y control cognitivo) (Heeren y Philippot, 2010) (Semple, 2010), puede producir cambios en la actividad de los circuitos fronto-estriados (Chiesa y Serretti, 2009; Kilpatrick et al. 2011; Tang et al.2010), alterar los niveles de neurotransmisores (dopamina) (Kjaer et al. 2002), modular los patrones electroencefalográficos (Davidson et al. 2003; Lutz, Greischar, Rawlings, Ricard y Davidson, 2004) y producir un aumento del grosor cortical (Lazar et al. 2005). El objetivo de la presente revisión es investigar la evidencia de los tratamientos basados en mindfulness para personas (niños y adultos) con TDAH. 


\section{Métodos}

Se realizó una búsqueda en la base de datos electrónica Pubmed para identificar artículos que estudiaran tratamientos basados en mindfulness para personas con TDAH. Las palabras clave utilizadas fueron "mindfulness", "ADHD", "Attention Deficit Disorder with Hyperactivity" y las diferentes combinaciones de estos términos. El criterio de inclusión consistió en seleccionar artículos en inglés que emplearan intervenciones (individuales o grupales) basadas en mindfulness para tratar pacientes (niños y adultos) con TDAH. Se consideraron como intervenciones basadas en mindfulness tanto la reducción del estrés basada en atención plena (REBAP, en inglés Mindfulness-based Cognitive Therapy, MBCT) como la terapia cognitiva basada en mindfulness (TCBM, en inglés Mindfulness-based Cognitive Therapy, MBCT).

La búsqueda no se limitó con ningún criterio temporal ni diseño experimental. No se incluyeron revisiones ni estudios de disertación. Esta búsqueda se realizó el 1 de septiembre de 2015.

Siete publicaciones cumplieron los criterios de selección para formar parte de la muestra final de la revisión (se encuentran resumidas en la tabla 1). Se trata de estudios homogéneos en cuanto a la intervención, el diseño utilizado y los resultados obtenidos. Las diferencias más significativas entre artículos hacen referencia a la muestra (niños o adultos) y las variables dependientes (diferentes cuestionarios autoinformados sobre TDAH, tareas atencionales o neuropsicológicas, entre otros).

\section{Resultados}

En el presente apartado se realiza una descripción de los diferentes estudios seleccionados. La estructura a seguir viene dada por la muestra seleccionada (niños o adultos, excepto el primer artículo que tiene participantes de las dos franjas de edad) y por el año de publicación. Este orden en la presentación coincide con la complejidad de los diseños de los estudios.

Sin embargo, antes de analizar los diferentes artículos es necesario resumir brevemente la temática central de la revisión: las intervenciones basadas en mindfulness para personas con TDAH. Estas intervenciones se han convertido en una nueva herramienta para modular o reducir el estrés y tratar trastornos psiquiátricos (Baer, 2003). Se trata de una intervención innovadora por su formato, basada en la meditación, que pone el énfasis en la observación y una postura no reactiva hacia los pensamientos, emociones y estados corporales de uno mismo. Aunque la relajación es un componente importante del mindfulness, el elemento central de la intervención es un proceso cognitivo intencional caracterizado por la autorregulación de la atención hacia el momento presente con una orientación de apertura y aceptación de las propias experiencias (Bishop et al., 2004).

La incorporación de esta técnica junto con otros métodos, particularmente la terapia cognitivo conductual, ha supuesto el desarrollo de diferentes tratamientos 


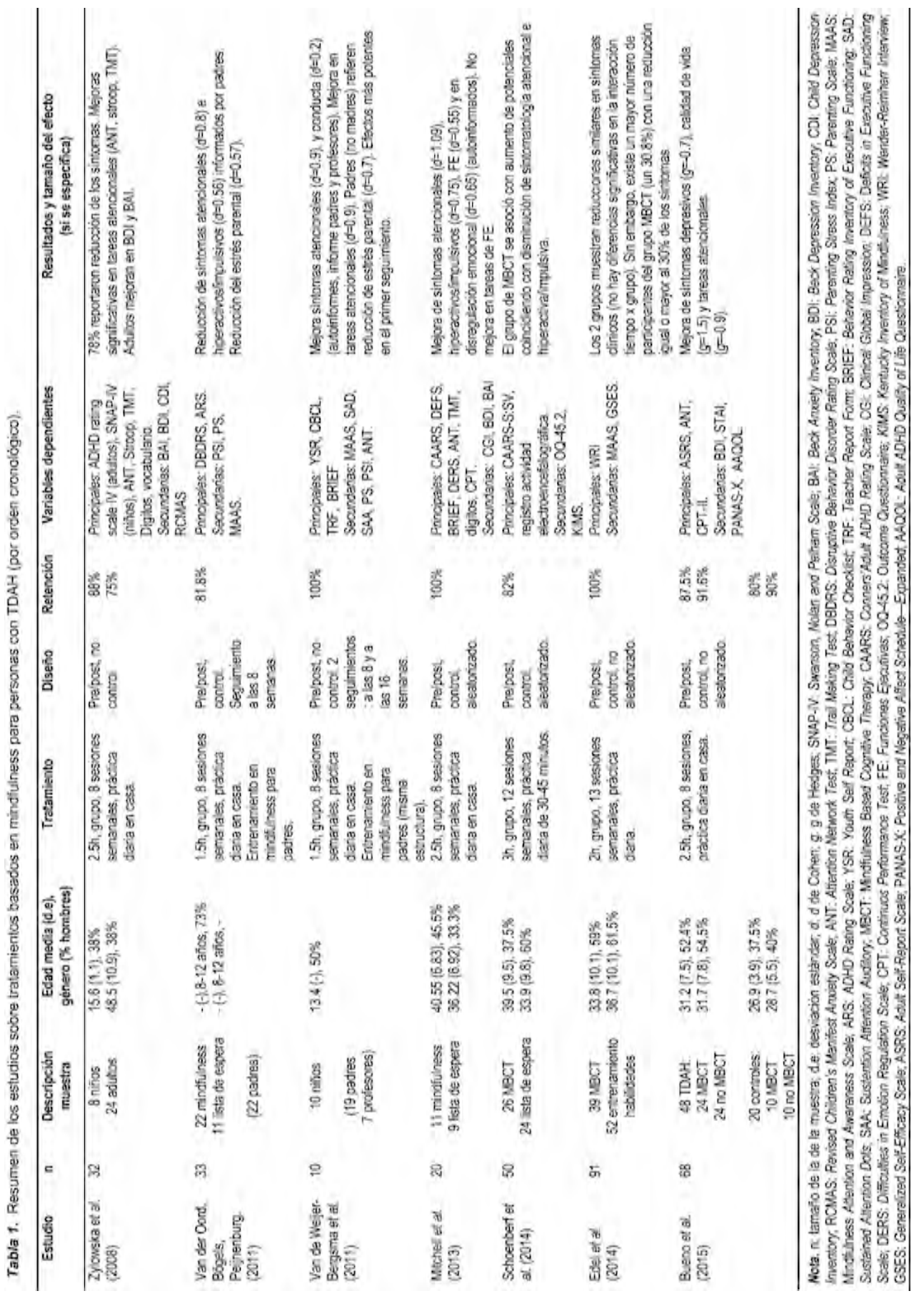


basados en mindfulness para el estrés, depresión, trastorno de personalidad límite, trastornos de ansiedad y abuso de sustancias (Baer, 2003) (Seidman, 2006).

No obstante, la investigación en cuanto a la eficacia de estos tratamientos para intervenir en el TDAH es mucho menor. Los pacientes con TDAH presentan déficits de control ejecutivo, incluyendo atención, memoria de trabajo, inhibición, motivación y regulación emocional (Nigg y Casey, 2005). Muchas de estas dificultades se pueden categorizar como déficits de autoregulación (Barkley, 1997). Las intervenciones basadas en mindfulness incrementan la capacidad de autoregulación de la atención y la emoción (Teasdale, Segal y Williams, 1995). El entrenamiento mediante este tipo de meditación puede incrementar la capacidad de alerta, orientación de la atención, metacognición, inhibición, memoria de trabajo así como la regulación emocional (Brown y Ryan, 2003). Estos argumentos, desde un punto de vista teórico, hacen que las intervenciones basadas en mindfulness se consideren un tratamiento prometedor para los síntomas nucleares del TDAH.

A continuación se muestra una selección de siete artículos que han intentado extrapolar a la práctica la idoneidad teórica de este tratamiento, con el objetivo de evaluar la eficacia de la intervención en pacientes con TDAH.

En el primero de los artículos, desarrollado por el equipo liderado por Zylowska et al. (2008), se puso a prueba un programa de entrenamiento en mindfulness de 8 sesiones semanales de 2.5 horas de duración con una muestra de 32 pacientes con TDAH ( 8 adolescentes y 24 adultos), con el objetivo de estudiar su eficacia y si era factible implementar un tratamiento de este tipo. Se trata de un diseño pre- test/post-test sin grupo control. La mayoría de los participantes completaron el entrenamiento y refirieron elevados nivel de satisfacción con el mismo. Se detectaron mejoras significativas tanto para adolescentes como para adultos en los síntomas autoinformados de TDAH (en un 78\% de los participantes, de los cuales un $30 \%$ obtuvo una reducción de la sintomatología del $30 \%$ o más). En cuanto a las tareas neuropsicológicas, también hubo mejoras significativas en conflicto atencional (Attention Network Test, ANT y Stroop color-palabra) y en atención dividida (Trail Making Test A y B, TMT A y B) pero no para pruebas que medían la memoria de trabajo. Considerando el subgrupo de adultos, estos redujeron significativamente los síntomas autoinformados de depresión y ansiedad.

Por otra parte, el equipo de van der Oord (van der Oord, Bögels y Peijnenburg, 2011) estudió la eficacia de un tratamiento similar (de una duración inferior, 8 sesiones de 1.5 horas) en una muestra de 33 niños (pre/post, 22 niños en el grupo de tratamiento y 11 en el grupo control formado por la lista de espera, aleatorizados, con un seguimiento a las 8 semanas). Los padres de los 22 niños incluidos en el entrenamiento realizaron un tratamiento paralelo de las mismas características ( $\mathrm{n}=$ 22). En cuanto a los resultados, se detectó una reducción significativa de los síntomas atencionales e hiperactivos/impulsivos informados por los padres y una disminución de los síntomas de los propios progenitores. Por otra parte, los padres mostraron un aumento significativo de su conciencia de atención plena. También 
se analizaron los cambios entre las puntaciones pre y seguimiento a las 8 semanas, detectando reducciones significativas de la sintomatología atencional e hiperactiva/ impulsiva tanto en los niños como en los padres. Adicionalmente se observó una reducción del estrés paterno y de la sobrerreacción. El estudio también incorporó la evaluación de los profesores de los niños que no mostró reducciones significativas de la sintomatología.

En el mismo año el equipo de van de Weijs-Bergsma (van de Weijer-Bergsma, Formsma, de Bruin y Bögels, 2011) estudió la eficacia de un tratamiento basado en meditación mindfulness muy similar al descrito anteriormente, pero esta vez con 10 adolescentes. En este caso también se describe un entrenamiento en mindfulness para los padres $(\mathrm{n}=19)$, paralelo al de los hijos. Los profesores también participaban contestando algunos cuestionarios. El diseño consistía en un pre/post sin grupo control con dos seguimientos a las 8 y a las 16 semanas (en este último seguimiento sólo participaron los adolescentes). En cuanto a los resultados: (a) a nivel atencional, en el seguimiento a las 8 semanas, tanto los padres (y no las madres ni los profesores) como los adolescentes, informaron de una reducción significativa de los problemas atencionales; (b) considerando los problemas externalizantes, los padres informaron de una mejora significativa tras el tratamiento y en el seguimiento a las 8 semanas; (c) los problemas internalizantes no mostraron una reducción significativa.

Por otra parte, no se detectaron mejoras significativas en cuanto al funcionamiento ejecutivo ni en la conciencia de la capacidad de atención plena (autoinformados). Los padres (y no las madres) informaron de una reducción significativa en el estrés paterno (que se mantuvo en el seguimiento a las 8 semanas). Los adolescentes no informaron de cambios con respecto a su fatiga ni a sus sentimientos de felicidad. Por último, las pruebas informatizadas para medir la atención detectaron una reducción significativa de la velocidad de reacción y de las falsas alarmas que no se mantuvieron en los seguimientos.

Mitchell et al. (2013) y su equipo publicaron un artículo en el que pretendían estudiar un tratamiento grupal basado en mindfulness para adultos con TDAH. En concreto utilizaron una muestra de 20 pacientes (pre/post, 11 en el grupo de tratamiento y 9 en el grupo control formado por la lista de espera, aleatorizados). El tratamiento utilizado era muy similar al del primer artículo descrito ( 8 sesiones de 2.5 horas). En cuanto a los resultados, el grupo que recibió el tratamiento redujo significativamente la sintomatología atencional (autoinformada y observada clínicamente) comparado con el grupo control. También se observaron diferencias significativas entre los grupos en cuanto a la sintomatología hiperactiva/impulsiva. Un $63.6 \%$ de los pacientes del grupo de tratamiento exhibieron una reducción de la sintomatología (autoinformada) igual o superior al 30\%, comparado con el $0 \%$ del grupo control. Clínicamente, el porcentaje de pacientes que mostraron una reducción de estas dimensiones fue superior ( $81.8 \%$ para los síntomas atencionales y $72.7 \%$ para los síntomas hiperactivos/impulsivos).

En cuanto a las funciones ejecutivas se detectó una mejora significativa en las 
escalas autoinformadas y a nivel clínico. Sin embargo, esta diferencia no se observó en las tareas neuropsicológicas (ANT, TMT, dígitos y Continuos Performance Test, $\mathrm{CPT}$ ). Por último, con respecto a la disregulación emocional, se observó una mejora significativa de los síntomas autoinformados.

Schoenberg et al. (2014) y su equipo de la universidad de Radboud publicaron un estudio en el año 2014 con el objetivo de comprobar la eficacia de una terapia cognitiva grupal basada en mindfulness de 12 sesiones de 3 horas de duración. La muestra empleada estaba compuesta por 50 participantes adultos: 26 en el grupo de tratamiento, 24 en el grupo control (lista de espera), todos aleatorizados.

Una de las peculiaridades de este estudio es que para evaluar la eficacia del tratamiento midieron potenciales evocados. Considerando los resultados, los pacientes del grupo de tratamiento tuvieron un incremento significativo de las amplitudes de los potenciales Pe (relacionados con la prominencia de los errores) y NoGo-P3 (relacionados con el control inhibitorio). Por otra parte, examinando los síntomas autoinformados se detectaron reducciones significativas con respecto a la sintomatología atencional, hiperactiva/impulsiva, malestar por los síntomas y rol social. Se observó un incremento significativo de todas las habilidades de atención plena autoinformadas. Por último, se detectó una correlación entre el incremento de la amplitud de Pe y una mejora de la sintomatología atencional así como entre el incremento de la amplitud de Nogo- P3 y una atenuación de la sintomatología hiperactiva/impulsiva.

Edel, Holter, Wassink y Juckel (2014) publicaron el único estudio que compara el entrenamiento en mindfulness con otra intervención psicológica. Con la muestra más amplia (91 participantes), compararon dos intervenciones grupales (entrenamiento en mindfulness y entrenamiento en habilidades derivado de la Terapia Dialéctica Conductual, los dos de 13 sesiones semanales de 2 horas de duración) para reducir síntomas del TDAH en pacientes adultos mayoritariamente medicados, que fueron asignados a una de las dos condiciones de manera no aleatorizada.

Los dos programas obtuvieron resultados similares en la reducción de los síntomas del TDAH, mejora de las habilidades de atención plena y de la autoeficacia (los tamaños del efecto fueron de rango bajo-medio). Sin embargo, un 30.8\% de los pacientes del grupo de mindfulness consiguió un decremento de los síntomas igual o superior al $30 \%$, mientras que en la otra condición experimental lo lograron solamente el 11.5\% (aunque estas diferencias no resultaron significativas).

Recientemente, Bueno y colaboradores (Bueno et al. 2015) de la Universidad de Sao Paulo, publicaron un artículo con el objetivo de valorar qué efectos tenía el entrenamiento en mindfulness en relación al estado de ánimo, la calidad de vida y la atención en una muestra de adultos con diagnóstico de TDAH comparado con un grupo control. La muestra de estudio era de 68 sujetos, 48 pacientes con diagnóstico de TDAH y 20 controles. Los pacientes y controles no fueron asignados aleatoriamente a la condición experimental. De los 48 pacientes, 24 accedieron a la intervención en atención plena, y de los 20 controles lo hicieron 10 . El entrenamien- 
to en mindfulness consistió en 8 sesiones semanales con una duración de 2.5 horas. La formación contemplaba también el entrenamiento entre sesiones en el que los sujetos tenían que practicar diariamente tanto de manera formal como informal. Para facilitar esta tarea, al inicio del programa se les entregó material con diferentes ejercicios de mindfulness. En cuanto a los resultados, se observó que los pacientes con TDAH obtuvieron peores puntuaciones en todas las medidas empleadas respecto al grupo control (personas sanas). Los resultados en relación a la intervención con mindfulness, demostraron una mejoría en el estado de ánimo, la calidad de vida, la atención sostenida y el control ejecutivo en ambos grupos, pacientes con TDAH y controles, alcanzando tamaños del efecto grandes.

\section{Discusión}

Como se ha podido observar, los siete artículos seleccionados aportan resultados favorables en referencia a la aplicación de intervenciones basadas en mindfulness para TDAH. No obstante, antes de proceder a interpretar los resultados y a extraer conclusiones es necesario hacer una descripción general de la metodología seguida por los artículos.

Fijándonos en los siete estudios, podemos observar que un $71 \%$ incluye un grupo control (en la mayoría de los casos formado por la lista de espera). Sólo 2 de los 7 artículos hacen seguimientos de los pacientes (un estudio a 8 semanas y el otro hasta 16 semanas). El tamaño de las muestras oscila entre los 10 y los 91 participantes, con una muestra media de 43 participantes. En cuanto al tratamiento, las intervenciones basadas en mindfulness tienen una estructura bastante similar; 5 de los estudios realizan tratamientos de 8 sesiones (los otros 2 utilizan programas de 12 y 13 sesiones). Dos de los estudios tienen una duración por sesión de 1.5 horas y los otros cinco artículos describen duraciones entre 2 y 3 horas. La retención de los participantes en el tratamiento es bastante elevada en todos los casos, variando del 75 al 100\%. Por último, es importante considerar que todos los estudios han realizado intervenciones grupales.

Si examinamos los resultados de manera global, podemos afirmar que todos los estudios obtienen mejoras en la sintomatología atencional e hiperactiva/ impulsiva después de aplicar el tratamiento, en varios casos con reducciones superiores al 30\% de la sintomatología. Estos resultados suelen hacer referencia a cuestionarios autoinformados (o informados por los padres y/o profesores en el caso de los niños), siendo éste uno de los puntos débiles en este ámbito de investigación. Y es que sólo 2 de los artículos evalúa la sintomatología mediante la exploración clínica.

Otra manera de explorar los síntomas asociados al TDAH es a través de tareas o experimentos de laboratorio que reflejen los déficits que conlleva el trastorno (problemas atencionales y de control ejecutivo). En este caso los resultados no son tan favorables. A pesar de ello, algunos estudios han obtenido discretas mejoras (poco consistentes entre los distintos artículos) en tareas de laboratorio que evalúan 
funciones atencionales y ejecutivas (subtests de la ANT, Stroop, TMT A y B, CPTII). Por otro lado, el estudio de Schoenberf et al. (2014) refleja cambios favorables en los potenciales evocados después del tratamiento.

Otra crítica importante de la muestra de artículos seleccionados es el control que se hace de los tratamientos farmacológicos que toman los participantes. Mientras que algunos de los estudios ni siquiera recogen esta información, otros se limitan a aceptar pacientes con dosis estables de un fármaco y a excluir aquellos que sufren modificaciones de la pauta farmacológica durante el tratamiento.

Siguiendo con la metodología de los estudios, es necesario apuntar que se trata de diseños muy sencillos con muestras pequeñas (pre/post, con grupo control en el mejor de los casos). En la mayoría de los estudios toman como referencia los participantes en lista de espera, siendo necesario para futuras investigaciones contrastar el tratamiento basado en mindfulness con otras intervenciones (psicológicas y farmacológicas). En este sentido, uno de los aspectos a mejorar es el del seguimiento de los efectos del tratamiento. Se trata de un aspecto que sólo se incorpora en dos de los estudios, llegando sólo a las 16 semanas de seguimiento.

Por último, y haciendo referencia a las limitaciones de la presente revisión, un aspecto a desarrollar sería el de cuantificar la potencia de los diferentes estudios (mediante el tamaño del efecto, por ejemplo) para poder identificar los resultados más importantes, establecer las diferencias entre artículos y realizar una revisión sistemática.

Como conclusión, los estudios anteriores han mostrado resultados aún preliminares pero prometedores. Con el objetivo de aportar un sólido cuerpo de conocimiento en esta línea de trabajo será necesario incorporar ciertas mejoras, tales como conseguir muestras más amplias, grupos controles (participantes que sigan otros tratamientos, no sólo en lista de espera), incorporar autoinformes de familiares y profesores y evaluaciones clínicas, incluir pruebas neuropsicológicas y tareas de laboratorio (que midan no sólo síntomas atencionales sino déficits en memoria de trabajo y disregulación emocional) y examinar los efectos a largo plazo del entrenamiento en mindfulness.

\section{Referencias bibliográficas}

Baer, R. (2003). Mindfulness Training as a Clinical Intervention: A Conceptual and Empirical Review. Clinical Psychology: Science And Practice, 10, 125-143.

Barkley, R. (1997). Attention-Deficit/Hyperactivity Disorder, Self-Regulation, and Time. Journal OfDevelopmental \& Behavioral Pediatrics, 18, 271-279.

Biederman, J. (2004). Impact of comorbidity in adults with attentiondeficit/hyperactivity disorder. Journal of Clinical Psychiatry, 65, 3-7.

Bishop, S., Lau, M., Shapiro, S., Carlson, L., Anderson, N., y Carmody, J. et al. (2004). Mindfulness: A Proposed Operational Definition. Clinical Psychology: Science And Practice, 11, 230-241.

Brown, K., y Ryan, R. (2003). The benefits of being present: Mindfulness and its role in psychological well-being. Journal Of Personality And Social Psychology, 84, 822-848. 
Bueno, V., Kozasa, E., da Silva, M., Alves, T., Louzã, M., y Pompéia, S. (2015). Mindfulness Meditation Improves Mood, Quality of Life, and Attention in Adults with Attention Deficit Hyperactivity Disorder. Biomed Research International, 2015, 1-14. doi:10.1155/2015/962857

Chiesa, A., y Serretti, A. (2009). A systematic review of neurobiological and clinical features of mindfulness meditations. Psychological Medicine, 40, 1239-1252.

Davidson, R., Kabat-Zinn, J., Schumacher, J., Rosenkranz, M., Muller, D., y Santorelli, S. et al. (2003). Alterations in Brain and Immune Function Produced by Mindfulness Meditation. Psychosomatic Medicine, 65, 564-570.

Edel, M., Holter, T., Wassink, K., y Juckel, G. (2014). A Comparison of Mindfulness- Based Group Training and Skills Group Training in Adults With ADHD: An Open Study. Journal Of Attention Disorders.

Heeren, A., y Philippot, P. (2010). Changes in Ruminative Thinking Mediate the Clinical Benefits of Mindfulness: Preliminary Findings. Mindfulness, 2, 8-13. Hofmann, S., Sawyer, A., Witt, A., y Oh, D. (2010). The effect of mindfulness-based therapy on anxiety and depression: A meta-analytic review. Journal Of Consulting And Clinical Psychology, 78, 169-183.

Jha, A., Krompinger, J., y Baime, M. (2007). Mindfulness training modifies subsystems of attention. Cognitive, Affective, \& Behavioral Neuroscience, 7, 109-119.

Kabat-Zinn, J. (2003). Mindfulness-Based Interventions in Context: Past, Present, and Future. Clinical Psychology: Science And Practice, 10, 144-156.

Kilpatrick, L., Suyenobu, B., Smith, S., Bueller, J., Goodman, T., y Creswell, J. et al. (2011). Impact of mindfulnessbased stress reduction training on intrinsic brain connectivity. Neuroimage, 56, 290-298.

Kjaer, T., Bertelsen, C., Piccini, P., Brooks, D., Alving, J., y Lou, H. (2002). Increased dopamine tone during meditation-induced change of consciousness. Cognitive Brain Research, 13, 255-259.

Lazar, S., Kerr, C., Wasserman, R., Gray, J., Greve, D., y Treadway, M. et al. (2005). Meditation experience is associated with increased cortical thickness. Neuroreport, 16, 1893-1897.

Lutz, A., Greischar, L., Rawlings, N., Ricard, M., y Davidson, R. (2004). Long-term meditators self-induce highamplitude gamma synchrony during mental practice. Proceedings Of The National Academy Of Sciences, $101,16369-16373$.

Marije Boonstra, A., Oosterlaan, J., Sergeant, J., y Buitelaar, J. (2005). Executive functioning in adult ADHD: a meta-analytic review. Psychological Medicine, 35, 1097-1108.

Martel, M. (2009). Research Review: A new perspective on attention- deficit/hyperactivity disorder: emotion dysregulation and trait models. Journal Of Child Psychology And Psychiatry, 50, 1042-1051.

Mitchell, J., McIntyre, E., English, J., Dennis, M., Beckham, J., y Kollins, S. (2013). A Pilot Trial of Mindfulness Meditation Training for ADHD in Adulthood: Impact on Core Symptoms, Executive Functioning, and Emotion Dysregulation. Journal Of Attention Disorders.

Nigg, J., y Casey, B. (2005). An integrative theory of attention-deficit/ hyperactivity disorder based on the cognitive and affective neurosciences. Develop. Psychopathol., 17.

Schachter, H. M., Pham, B., King, J., Langford, S. y Moher, D. (2001). How efficacious and safe is short-acting methylphenidate for the treatment of attention-deficit hyperactivity disorder in children and adolescents? A meta-analysis. Canadian Medical Association Journal, 165, 1475-1488.

Schoenberg, P., Hepark, S., Kan, C., Barendregt, H., Buitelaar, J., y Speckens, A. (2014). Effects of mindfulnessbased cognitive therapy on neurophysiological correlates of performance monitoring in adult attentiondeficit/hyperactivity disorder. Clinical Neurophysiology, 125, 1407-1416.

Seidman, L. (2006). Neuropsychological functioning in people with ADHD across the lifespan. Clinical Psychology Review, 26, 466-485.

Semple, R. (2010). Does Mindfulness Meditation Enhance Attention? A Randomized Controlled Trial. Mindfulness, 1, 121-130. Tang, Y., Lu, Q., Geng, X., Stein, E., Yang, Y., y Posner, M. (2010). Short-term meditation induces white matter changes in the anterior cingulate. Proceedings Of The National Academy OfSciences, $107,15649-15652$.

Teasdale, J., Segal, Z., y Williams, J. (1995). How does cognitive therapy prevent depressive relapse and why should attentional control (mindfulness) training help?. Behaviour Research And Therapy, 33, 25-39.

van de Weijer-Bergsma, E., Formsma, A., de Bruin, E., y Bögels, S. (2011). The Effectiveness of Mindfulness Training on Behavioral Problems and Attentional Functioning in Adolescents with ADHD. Journal Of Child And Family Studies, 21, 775-787.

van der Oord, S., Bögels, S., y Peijnenburg, D. (2011). The Effectiveness of Mindfulness Training for Children with ADHD and Mindful Parenting for their Parents. Journal Of Child And Family Studies, 21, 139-147.

Zylowska, L., Ackerman, D., Yang, M., Futrell, J., Horton, N., y Hale, T. et al. (2007). Mindfulness Meditation Training in Adults and Adolescents With ADHD: A Feasibility Study. Journal Of Attention Disorders, 11, 737-746. 